OPEN ACCESS

Edited by:

Shuyu Zhang,

Sichuan University, China

Reviewed by:

Yunliang Zhang,

National Institutes of Health (NIH),

United States

Handu Liu,

Macau University of Science and

Technology, Macao SAR, China

*Correspondence:

Baixue Li

baixuelee@163.com

tThese authors have contributed equally to this work

Specialty section:

This article was submitted to Molecular and Cellular Oncology, a section of the journal

Frontiers in Cell and Developmental

Biology

Received: 14 August 2021 Accepted: 10 November 2021 Published: 02 December 2021

Citation:

Wu P, Qiao L, Yu H, Ming H, Liu C, Wu $W$ and Li B (2021) Arbutin

Alleviates the Liver Injury of a-Naphthylisothiocyanate-induced Cholestasis Through Farnesoid X Receptor Activation. Front. Cell Dev. Biol. 9:758632. doi: 10.3389/fcell.2021.758632

\section{Arbutin Alleviates the Liver Injury of $\alpha$-Naphthylisothiocyanate-induced Cholestasis Through Farnesoid X Receptor Activation}

\author{
Peijie $\mathrm{Wu}^{\dagger}$, Ling $\mathrm{Qiao}^{\dagger}$, Han $\mathrm{Yu}^{\dagger}$, Hui Ming ${ }^{\dagger}$, Chao Liu, Wenjun Wu and Baixue $\mathrm{Li}^{*}$ \\ School of Basic Medical Sciences, Chengdu University of Traditional Chinese Medicine, Chengdu, China
}

Cholestasis is a kind of stressful syndrome along with liver toxicity, which has been demonstrated to be related to fibrosis, cirrhosis, even cholangiocellular or hepatocellular carcinomas. Cholestasis usually caused by the dysregulated metabolism of bile acids that possess high cellular toxicity and synthesized by cholesterol in the liver to undergo enterohepatic circulation. In cholestasis, the accumulation of bile acids in the liver causes biliary and hepatocyte injury, oxidative stress, and inflammation. The farnesoid $X$ receptor (FXR) is regarded as a bile acid-activated receptor that regulates a network of genes involved in bile acid metabolism, providing a new therapeutic target to treat cholestatic diseases. Arbutin is a glycosylated hydroquinone isolated from medicinal plants in the genus Arctostaphylos, which has a variety of potentially pharmacological properties, such as anti-inflammatory, antihyperlipidemic, antiviral, antihyperglycemic, and antioxidant activity. However, the mechanistic contributions of arbutin to alleviate liver injury of cholestasis, especially its role on bile acid homeostasis via nuclear receptors, have not been fully elucidated. In this study, we demonstrate that arbutin has a protective effect on $\alpha$-naphthylisothiocyanate-induced cholestasis via upregulation of the levels of FXR and downstream enzymes associated with bile acid homeostasis such as Bsep, Ntcp, and Sult2a1, as well as Ugt1a1. Furthermore, the regulation of these functional proteins related to bile acid homeostasis by arbutin could be alleviated by FXR silencing in L-02 cells. In conclusion, a protective effect could be supported by arbutin to alleviate ANIT-induced cholestatic liver toxicity, which was partly through the FXR pathway, suggesting arbutin may be a potential chemical molecule for the cholestatic disease.

\section{Keywords: cholestasis, liver injury, arbutin, farnesoid X receptor, $\alpha$-naphthylisothiocyanate, bile acid metabolism,} ursodeoxycholic acid

\section{INTRODUCTION}

Cholestasis, a kind of stressful syndrome induced by hormones, drugs, cytokines, or progressive bile duct destruction or stones, increases the probability of hepatitis, cirrhosis, liver cancer, or other gallbladder and hepatic diseases due to the excessive accumulation of toxic biliary components like cholesterol, bilirubin, and bile acids (BAs) in the liver and blood (Zollner and Trauner, 2006; Li et al., 2016). Intrahepatic cholestasis has been divided into intrahepatic or drug-induced or inflammatory cholestasis, as well as primary biliary cirrhosis (Zollner and Trauner, 2008; Li et al., 2020b). Upon the 
stress of toxic biliary, the cholestatic diseases will lead to hepatocyte apoptosis, necrosis, jaundice, hypercholesterolemia, cirrhosis, fibrosis, liver failure, even cholangiocellular or hepatocellular carcinomas, and ultimately life-threatening (Hirschfield et al., 2013; Feldman and Sokol, 2020). Recent studies also reported that the incidence of liver cancer, biliary tract cancer, and several other gastrointestinal tumors is increased when the homeostasis of bile acids metabolism is disrupted (Navaneethan et al., 2021; Ocvirk and O'Keefe, 2021; Rimland et al., 2021; Wu et al., 2021). Disruption of bile acid metabolic homeostasis including dysfunction of BA synthesis, obstruction of the bile duct, and impaired secretion by cholangiocytes will further exacerbate cholestasis (Nakanishi and Saxena, 2015; Keitel et al., 2019). Accordingly, proper recuperation of bile acids is paramount for cholestasis therapy and prevention.

$\alpha$-Naphthylisothiocyanate (ANIT) is commonly applied to induce cholestatic disease in vivo and in vitro for toxicological studies (Mariotti et al., 2018). Recent studies also suggested the effect of ANIT on hepatocytes by directly destroying bile duct epithelial cells (BECs) (Carino et al., 2020; López-Riera et al., 2020). The excessive accumulation of BAs inside hepatic cells is the primary reason for hepatic damage related to cholestasis. Hepatocytes have two special polarity domains distinguished as basolateral and bile canalicular domains, which are localized with tight junction proteins and specific bile acid (BA) transporters ( $\mathrm{Li}$ et al., 2016). In general, BAs are produced by cholesterol. Bile canalicular domains containing several canalicular efflux transporters, such as multidrug resistance-associated protein 2 (Mrp 2) and bile salt export pump (Bsep), regulate the transportation of BAs from hepatocytes to the bile canalicular domain for BA generation (Li et al., 2016). While basolateral domains containing organic anion transporter 2 (Oatp2) and $\mathrm{Na}^{+}$-dependent taurocholate cotransporter (Ntcp) regulate a primary step in reabsorption of bile acid from portal venous blood to hepatocytes. Besides, other basolateral export transporters, like Mrp-3 and Mrp-4, transport BAs from liver cells to portal blood (Meier and Stieger, 2002; Alrefai and Gill, 2007; Halilbasic et al., 2013; Li et al., 2019). Additionally, a series of bile acid synthetic enzymes containing Cyp8b1 (oxysterol $12 \alpha$ hydroxylase) and cholesterol $7 \alpha$-hydroxylase (Cyp7a1) and metabolizing enzymes like sulfate transferase 2 (SULT2a1) or UDP glucuronosyltransferase 1 (UGT1a1) have also been reported to control the bile acid homeostasis (Thakare et al., 2018). Accordingly, these impaired BA transporters or genes involved in BA metabolisms have been reported to be related to cholestasis. Recently, extensive studies have shown that these aforementioned factors were controlled by specific nuclear receptors (NR) containing the farnesoid $\mathrm{X}$ receptor (FXR) signaling pathway. The FXR was reported as the first nuclear receptor of bile acid endogenous ligands, which has been suggested to regulate the bile acid homeostasis and metabolism of cholestasis. Mechanistically, the FXR, heterodimerized with RXR, promoted small heterodimer partner (SHP) binding with BA efflux transporters and enzymes (Stofan and Guo, 2020). Similarly, the FXR-null mice showed increased bile acid-related hepatocyte toxicity and alkaline phosphatase (AP) levels, or decreased expression of
BA enzymes and transporters related to cholestasis ( $\mathrm{Li}$ and Chiang, 2020). Therefore, inhibition of the FXR would aggravate the cholestatic injury; activation of the FXR might be a critical therapeutic target for cholestasis therapy. Ursodeoxycholic acid (UDCA) has been applied for anticholestasis drug in clinical trials by the Food and Drug administration (FDA) (Corpechot et al., 2020; Dyson and Jones, 2020). While, more than $40 \%$ of patients with primary biliary cholangitis (PBC) cannot be treated adequately and also have serious complications (Dyson and Jones, 2020; Kumar and Kulkarni, 2020). Obeticholic acid (OCA), which could activate the FXR signaling pathway, has been recently approved for PBC by the FDA. However, there are no other proper drugs for cholestatic disease (Kjærgaard et al., 2021). Accordingly, the development of more effective strategies for cholestasis may be imperative. Intriguingly, recent studies implied that some natural products such as picroside and yangonin also have protective effects against cholestasis via the FXR pathway (Dong et al., 2019; Keitel et al., 2019; Li et al., 2020a). Thus, focusing on the FXR pathway may be a promising therapy for liver disease caused by cholestasis.

Arbutin ( $\beta$-d-glucopyranoside of hydroquinone, molecular formula: $\left.\mathrm{C}_{12} \mathrm{H}_{16} \mathrm{O}_{7}\right)$, a natural hydroquinone glycoside (Figure 1A) present in leaves and fruits of various plants, such as Arctostaphylos uva-ursi belonging to Ericaceae and Saxifragaceae families, is often used for various skin diseases (Xu et al., 2015; Garcia-Jimenez et al., 2017; Zhou et al., 2019). Leaves or fruits of these plants have been used as traditional medicines for wound healing and urinary tract infections for hundreds or even thousands of years by the aborigines of the American continents and China (Lindpaintner, 1939; Xu et al., 2015; Garcia-Jimenez et al., 2017). And all this time, arbutin has been applied to possess anti-inflammatory, anti-apoptosis, antimicrobial, and antioxidant effects. Even today, arbutin has also been used for the treatment of asthma (Migas and KrauzeBaranowska, 2015; Ortiz-Ruiz et al., 2015; Tang and Chen, 2015). Due to its mildly therapeutic properties, arbutin is also widely used in food, health-care, and cosmetic industries. Previous studies suggested that arbutin may prominently mitigate liver damage caused by $\mathrm{CCl}_{4}$ (carbon tetrachloride), radiation, lipopolysaccharides (LPSs), and D-galactosamine (D-GalN) (Mirshahvalad et al., 2016; Nadi et al., 2019; Jurica et al., 2020). But there have been limited research studies on the potential effect of arbutin against cholestatic liver disease and its fundamental mechanisms. In this study, we demonstrated that arbutin can attenuate the injury in ANIT-induced cholestasis, partly via FXR pathway activation.

\section{MATERIALS AND METHODS}

\section{Chemical Reagents}

Arbutin (more than 98\% purity) wad supplied by Aladdin (Shanghai, China). ANIT (more than 98\% purity) was purchased from Sigma. UDCA was purchased from the Aladdin (Shanghai, China). Ntcp, Bsep, FXR, and Cyp7a1 as well as GAPDH antibody were from Beyotime Biotech (Beyotime, 
A<smiles>OC[C@H]1O[C@@H](Oc2ccc(O)cc2)[C@H](O)[C@H](O)[C@@H]1O</smiles>

C

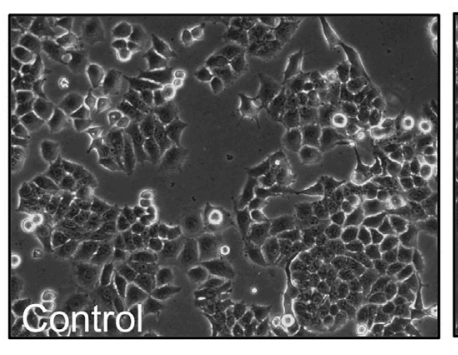

D

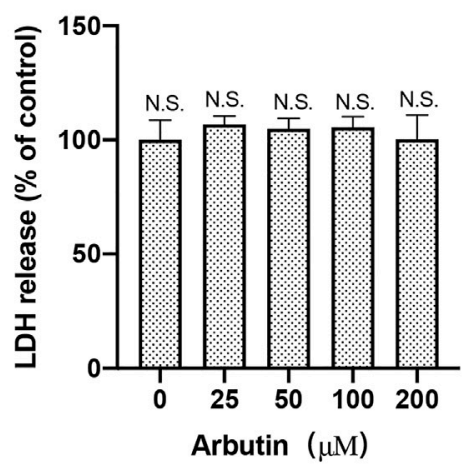

F

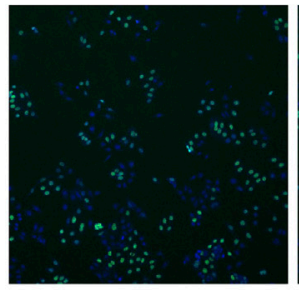

Arbutin 0

$(\mu \mathrm{M})$

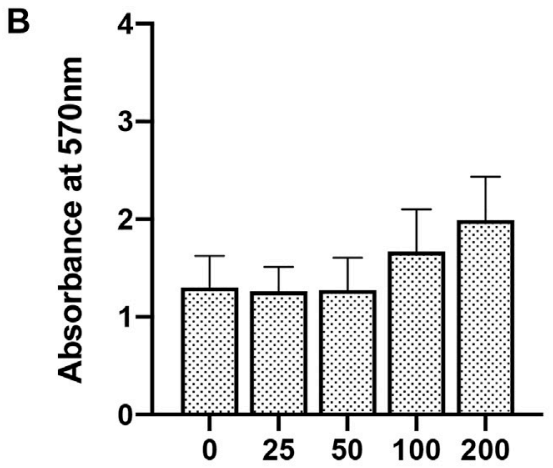

Arbutin $(\mu \mathrm{M})$

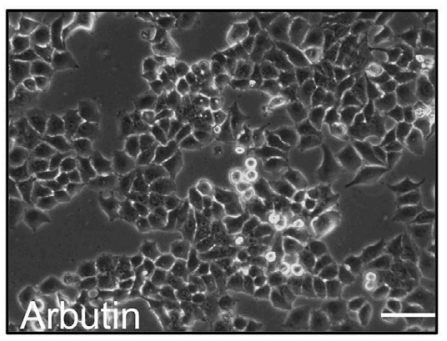

E

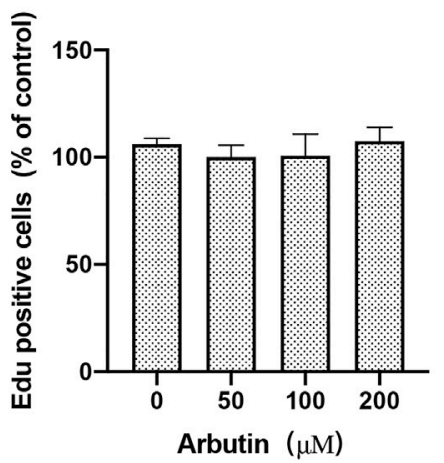

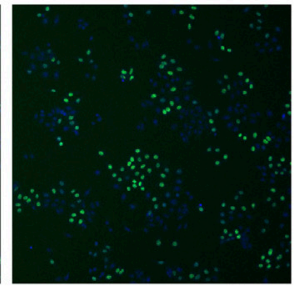

200

FIGURE 1| Arbutin has no significant cytotoxic effects on hepatocytes. Effects of arbutin on survival viability and morphology of cells. (A) The structure for arbutin. (B) Cell viability with or without arbutin treatment was measure by MTT assay. (C) Morphologies of cells treated with arbutin for $24 \mathrm{~h}$ (D) LDH assay was performed with arbutin treatment in L-02 cells. (E) Cell proliferation was detected by EdU incorporation assay. (F) Proliferation was measured by EdU assay. Representative images are shown. 
TABLE 1 | Experimental groups and treatment.

\begin{tabular}{|c|c|}
\hline Group & Treatment \\
\hline Control group & Mouse with the same concentrate vehicle buffer \\
\hline UDCA + ANIT group & Mouse with UDCA every day and ANIT \\
\hline ANIT group & Mouse with ANIT (75 mg/kg) \\
\hline Arbutin (low) + ANIT group & $\begin{array}{l}\text { Mouse with arbutin }(10 \mathrm{mg} / \mathrm{kg}) \text { every day and } \\
\text { ANIT }\end{array}$ \\
\hline $\begin{array}{l}\text { Arbutin (medium) }+ \text { ANIT } \\
\text { group }\end{array}$ & $\begin{array}{l}\text { Mouse with arbutin }(20 \mathrm{mg} / \mathrm{kg}) \text { every day and } \\
\text { ANIT }\end{array}$ \\
\hline Arbutin (high) + ANIT group & $\begin{array}{l}\text { Mouse with arbutin }(40 \mathrm{mg} / \mathrm{kg}) \text { every day and } \\
\text { ANIT }\end{array}$ \\
\hline
\end{tabular}

Shanghai, PR China) and Bioss Biotechnology (Bioss, Beijing, China). Goat anti-mouse IgG-HRP or goat anti-rabbit IgG-HRP were purchased from Santa Cruz Biotechnology (CA, United States). Immunofluorescence antibodies of Alexa Fluor were purchased from Invitrogen Life Science (CA, United States). siFXR ((5'-CAAGTGACCTCGACAACAA- $\left.3^{\prime}\right)$ was from Qingke Bioscience (Chengdu, China). Kits for determination of alkaline phosphatase (ALP; cat. no. A059-1), alanine transaminase (C0009-2), aspartate transaminase (C010-2), $\gamma$-glutamyltranspeptidase $(\gamma$-GT; cat. no. C017-1), total bile acids (TBA; cat. no. E003-1), and direct bilirubin (DBIL; cat. no. C019-2) were obtained from Nanjing Jiancheng. Other chemical reagents were of analytical grade.

\section{Animals}

Six- to seven-week-old adult C57BL/6 mice (male) were obtained from Dossy Bioscience (Chengdu, China). The animals were supported on a cycle with 12-hour light and 12-hour dark with temperature $\left(25 \pm 1^{\circ} \mathrm{C}\right)$ and $(46 \pm 5 \%)$ humidity for 1 week prior to experiments. The $\mathrm{C} 57 \mathrm{BL} / 6$ mice were distributed into six groups (eight mice in each group) randomly (Table 1). Chemical drug doses and abidance of exposure were based on previous research studies. Arbutin $(10,20$, and $40 \mathrm{mg} / \mathrm{kg}$ ) or $30 \mathrm{mg} / \mathrm{kg}$ UDCA or buffer alone was intraperitoneally (i.p.) administered to mice every day for 1 week. On the fifth experimental day, 3 hours after arbutin, UDCA, or vehicle treatment, mice were intragastrically (i.g.) administered $\alpha$-naphthylisothiocyanate (ANIT) with $75 \mathrm{mg} / \mathrm{kg}$. On the seventh day, all groups were euthanized and fasted to collect blood, bile, and livers for further analysis 4 hours after treatment with arbutin, UDCA, or vehicle buffer. The mice were intragastrically euthanized with $150 \mathrm{mg} / \mathrm{kg}$ pentobarbital following the guidelines of the Animal Ethics Committee. The liver, blood, gallbladder, and duodenum were immediately excised after euthanasia and were stored at $-80^{\circ} \mathrm{C}$. All animal studies were approved by the Animal Ethics Committee of Chengdu University of Traditional Chinese Medicine and performed following the regulations (Figure 2).

\section{Histopathology and Serum Biochemistry}

After euthanasia, the liver and other tissues were fixed with $4 \%$ polyoxymethylene, and the slices were excised from the anterior liver part of the left lobe, embedded in paraffin, and sectioned into $5-\mu \mathrm{m}$ sections. Each slide was stained with trichrome for hematoxylin and eosin (H\&E) and further intuitive liver histological assessment including hepatocellular necrosis, portal inflammation, or hyperplasia. Analysis of the surface areas of the hepatocytes was carried out under a high-resolution microscope (Olympus) with a photographic facility. The samples of blood stored at $4^{\circ} \mathrm{C}$ for 4 hours were centrifuged for 30 minutes at $12,000 \mathrm{rpm}$ to collect the supernatant. And ALP, $\gamma$-GT, ALT, TBIL, AST, and TBA of serum were measured using the Automatic Biochemistry Analyzer according to the manufacturers' instructions.

\section{Quantitative Analysis of Real-Time PCR}

Total RNAs were extracted using TRIzol reagent (Life technologies; no. 15596026) according to the manufacturers' instructions, and the quantity of total RNA was measured. Reverse transcription was performed to synthesis cDNA with BeyoRT $^{\mathrm{TM}}$ commercial Kit from Beyotime (Shanghai, China). As a template, cDNA was used for real-time PCR in triplicate with the SYBR Green Master Mix and specific primers on a Real-Time PCR System (Bio-Rad) (Table 2). GAPDH was normalized as an internal control to evaluate the efficiency.

\section{Cell Culture, Cell Viability Assays, and Transient Transfection Experiment}

Human hepatocyte (L-02) cells were cultured in DMEM containing $10 \%$ FBS, $100 \mathrm{U} / \mathrm{ml}$ antibiotics with streptomycin, penicillin, and streptomycin. The viability of L-02 cells was analyzed by MTT experiments. The cells were cultured with indicated concentrations of arbutin $(25,50,100$, and $200 \mu \mathrm{M})$ for 24 hours; vehicle groups were cultured with the same final concentration of DMSO (the final concentration must be $<0.001$ ). Then, MTT was added to each well to incubate for 3 hours at $37^{\circ} \mathrm{C}$. After discarding the supernatant, DMSO was used to dissolve the mixture, and absorbance was measured at a wavelength of $570 \mathrm{~nm}$ by the Microplate Reader system (BIO-RAD). For transient transfection experiment, L-02 cells were seeded with a proper density for 24 -well plates at $7 \times 10^{5}$ cells each well. A control siRNA was used as a negative control. Cells were transfected using Lipofectamine ${ }^{\mathrm{TM}} 3000$ (Thermo Fisher Scientific). These transfected cells were treated with drugs mentioned before for another 24 hours after transfection. Cell proliferation was detected by the EdU assay kit (RiboBio, C10310) according to the instruction completely. And images were obtained with a fluorescence microscope. For lactate dehydrogenase release (LDH) assay, cell cytotoxicity was assessed by a commercial kit from Beyotime Biotech (Beyotime, Shanghai, PR China). The experiment was carried out in accordance with the supplier's instructions.

\section{Protein Analyzed With Western Blot}

Total proteins of liver were lysate by RIPA buffer, and the concentration of protein samples were evaluated by a BCA Protein Assay Kit (Thermo Fisher) according to the instructions. Equivalent samples were subjected to SDS-PAGE and then transferred to the polyvinylidene difluoride (PVDF) membranes (Millipore Corporation, MA, United States). After sealing with $5 \%$ non-fat milk, the PVDF membranes were washed 


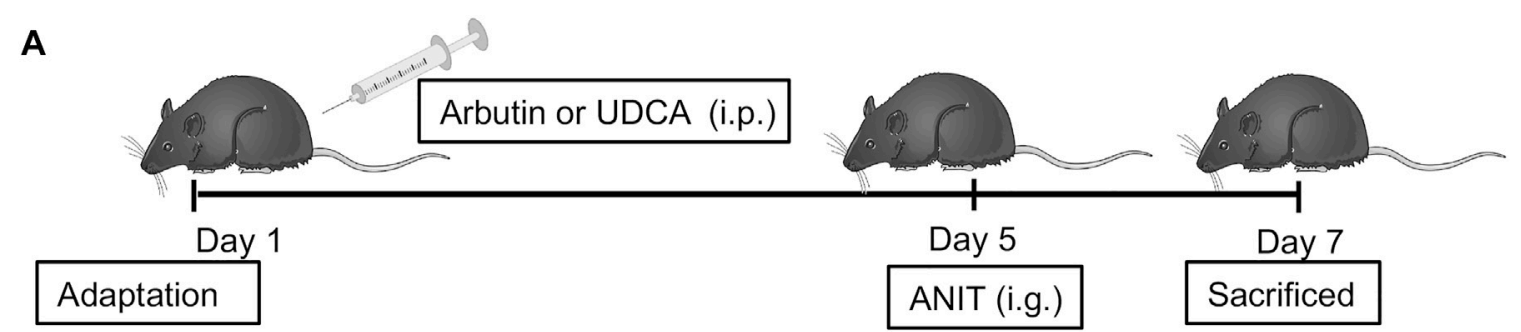

B

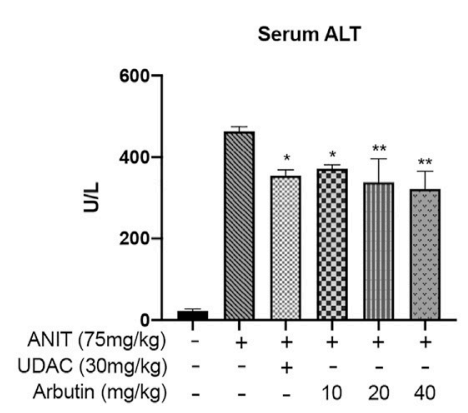

E
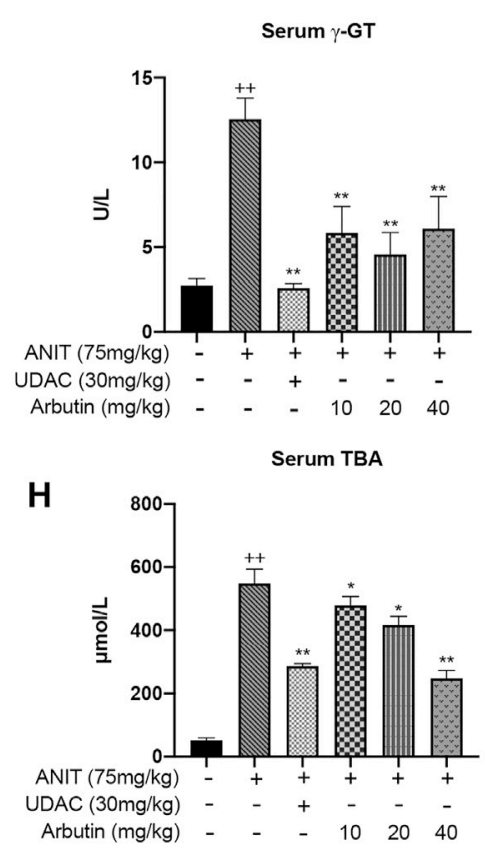

C

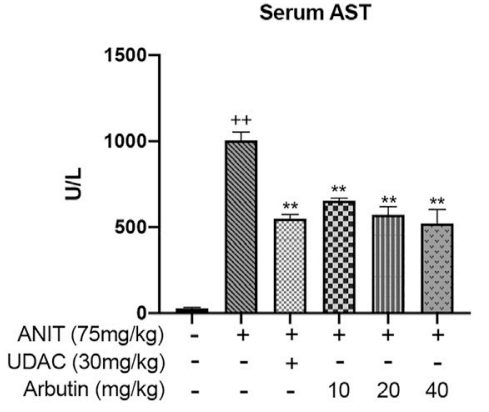

D

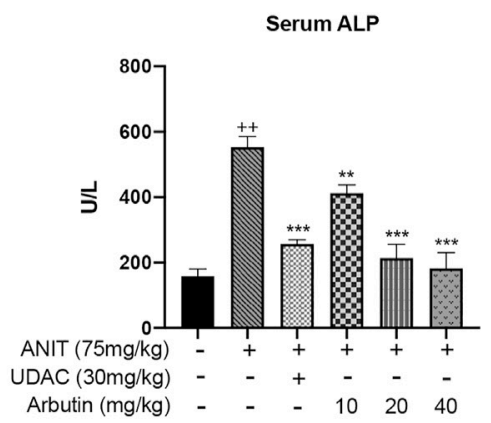

$\mathbf{F}$

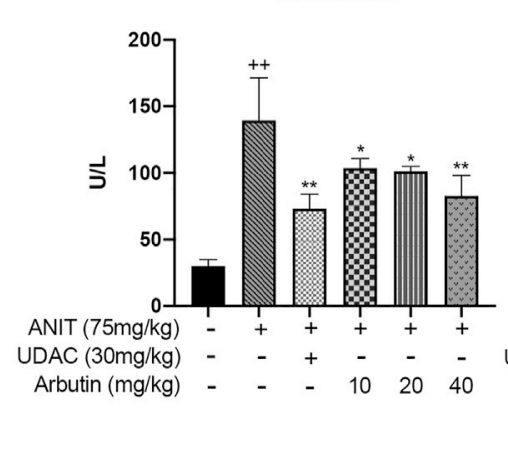

G

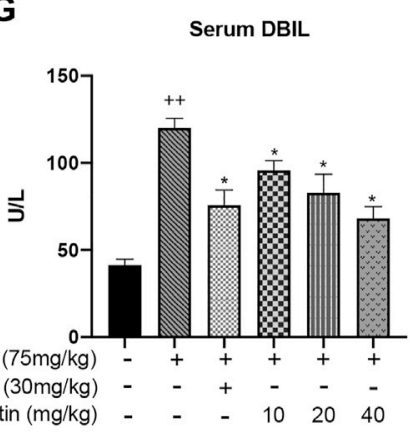

FIGURE 2| Hepatoprotective effects of arbutin related to cholestatic liver damage induced by ANIT. (A) The scheme for the animal experimental design. The serum biochemical indicators levels were elevated (B-H). Data are represented by mean $\pm \mathrm{SD}$. $(n=3)$. $p<0.05 ;+p<0.05$ vs. control; ${ }^{*} p<0.05$ vs. model.

and co-incubated with specific primary antibodies at $4^{\circ} \mathrm{C}$ overnight. Subsequently, the second antibody was incubated with the membrane for 1 hour at room temperature. Specific protein-antibody complexes were measured using enhanced chemiluminescence (ECL) reagents and collected with the gel imaging system (ChmiScope, Clinx, China).

\section{Immunofluorescence}

Cells were seeded on the glass cover slides (WHB scientific). After indicated treatment, cells were fixed in $4 \%$ formaldehyde, permeabilized by $0.3 \%$ Triton X-100, and blocked with $5 \%$ BSA. The slides were then incubated with indicated antibodies above at $4^{\circ} \mathrm{C}$ overnight and Alexa Fluor 488/594 antibodies (1:200 
TABLE 2 | Sequences of primer for qPCR analysis in this study.

\begin{tabular}{|c|c|c|}
\hline Gene & Forward 5'-3' & Reverse 5'-3' \\
\hline Ntcp & ATGGAGGCGCACAACGTATC & ACTACCAGAATGACGCTGAGC \\
\hline Bsep & GGACAATGATGTGCTTGTGG & CACACAAAGCCCCTACCAGT \\
\hline Oatp1b2 & GCACTGCGATGGATTCAGGAT & AGCTाTGGTCGGTGTAGCTTG \\
\hline MRP2 & GTGTGGATTCCCTTGGGCTाT & CACAACGAACACCTGCTTGG \\
\hline Ugt1a1 & CTGAGCCCTGCATCTATCTG & CCCAGAGGCGTTGACATA \\
\hline MRP3 & ACACCGAGCCAGCCATATAC & TCAGCTTCACATTGCCTGTC \\
\hline Cyp7a1 & GAACCTGTACATGAGGGAC & СТTCTTCAGAGGCTGCTाC \\
\hline Cyp8b1 & GTTGCAGCGTCTCTTCCAT & ССTTGСTCССTCAGAAACT \\
\hline GAPDH & ATGGAGAAGGCTGGGGCTCACCT & AGCCCTTCCACGATGCCAAAGTTGT \\
\hline
\end{tabular}

dilution) for 1 hour at room temperature. Nuclei were stained with 1:2000 diluted DAPI solution (Solarbio, C0060) at room temperature for 15 minutes, and the images were captured by using a DM2500 fluorescence microscope (Leica).

\section{Molecular Docking Study}

For molecular docking studies, the AutoDock Tools were used to identify the potential FXR agonists. The 3D structure file of NR1H4 (FXR, code: 4QE6) was downloaded from Protein Data Bank (Kudlinzki et al., 2019). The original structure of NR1H4 was then obtained by AutoDock Tools v1.5.6 to preserve charge and prepare pdbqt file for docking. Then, the hydrogenated SDF structures of arbutin were obtained from PubChem database. And, the structure with the lowest docking energy was measured by minimization. Finally, the resultant protein poses were ranked by scoring, and the image of top-ranked protein pose was further displayed by Discovery Studio Visualizer 2019 and PyMOL v1.8 software.

\section{Statistical Analysis}

All data were presented as mean $\pm \mathrm{SD}$. One-way analysis of variance (ANOVA) was used to analyze differences between multiple groups. And non-parametric Mann-Whitney U test and unpaired Student's t-test were used for two groups with GraphPad Prism software v8. As a threshold, $p<0.05$ was shown as statistically significant.

\section{RESULTS}

\section{Arbutin Has No Significant Cytotoxic Effects on Hepatocytes}

To investigate the cytotoxic of arbutin on hepatocyte cells, MTT assay of L-02 cells treated with arbutin was performed. The results suggested that $0-200 \mu \mathrm{M}$ of arbutin for 24 hours showed no obvious proliferative inhibition (Figures 1B,C). Moreover, the IC50 value of arbutin was 618.2 $\mu \mathrm{M}$ (Supplementary Figure S1A); Consistently, EdU incorporation assays and colony formation analysis confirmed that treatment with arbutin has no effects on the proliferation of hepatocyte cells (Figures 1E,F). The results also did not show enhanced cytotoxicity monitored via lactate dehydrogenase (LDH) assay (Figure 1D) Additionally, the serum levels of AST and ALT did not change significantly in mice treated with arbutin $40 \mathrm{mg} / \mathrm{kg}$ (data not shown), therefore implicating that arbutin did not exert significant cytotoxicity in vitro and in vivo. Accordingly, arbutin may have no significant cytotoxic effects on hepatocytes.

\section{Arbutin Alleviates the Hepatotoxicity and Cholestasis in ANIT-Treated Mice}

To evaluate the effects of arbutin on liver injury induced by cholestasis, C57BL/6 mice were treated with ANIT $(75 \mathrm{mg} / \mathrm{kg})$ to induce cholestasis. In brief, the vehicle and ANIT groups were treated with vacant solvent. The UDCA treatment with $30 \mathrm{mg} / \mathrm{kg}$ and several arbutin treatment groups were, respectively, pretreated with indicated drugs for 5 days. Then, all groups, except the control, were treated with ANIT that resolved in olive oil. Two days later, the serum and liver tissue samples were collected. Our results showed that the levels of ALT and AST in serum and the sensitive blood biochemical index of liver injury were significantly upregulated after ANIT treatment. The levels of TBIL, $\gamma$-GT, TBA, and ALP in serum, the key biochemical indicators relevant to liver damage, were also elevated by administration with ANIT (Figure 2). Accordingly, these results indicated that intrahepatic cholestatic liver damage had been successfully induced by ANIT. Notably, treatment with UDCA or arbutin visibly reduced the serum ALT and AST levels compared with ANIT treatment. Meanwhile, the levels of serum indicators in the UDCA- and arbutin-treated groups decreased significantly. Moreover, arbutin also reversed the liver injury induced by ANIT $(10,20$, and $40 \mathrm{mg} / \mathrm{kg}$ arbutin) in an adaptive response way $(p<0.01$ or $<0.05$ ) (Figure 2). Together, these data suggest that arbutin can alleviate the hepatotoxicity in ANIT-induced cholestasis in vivo.

\section{Arbutin Reverses the Morphologic Changes Caused by ANIT in Mice}

Morphological and histopathological data of liver were assessed to analyze the hepatoprotective effect of arbutin in vivo. The tissues acquired from the ANIT-treated mice displayed visible hepatic necrosis, inflammatory factor infiltration, and edema (Figure 3; Table 3). We also found that ANIT led to a gallbladder filling and a blackened color (data not shown). Congruously, treatment with UDCA and arbutin could significantly attenuate these pathological changes. Arbutin showed a hepatoprotective effect in an adaptive response way (Figure 3).

\section{Arbutin Alters the Expression of Proteins Related With Bile Acid Homeostasis}

In order to investigate the mechanism of arbutin in alleviating liver injury, the genes related to BA metabolism were measured 


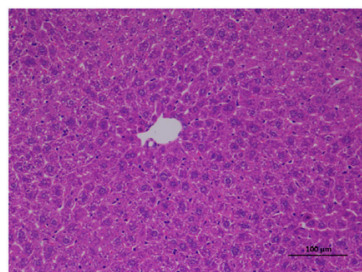

Control

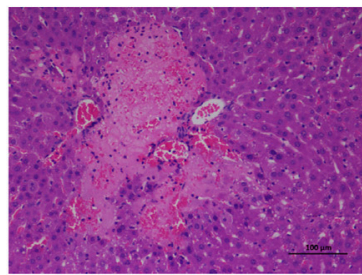

Arbutin $10 \mathrm{mg} / \mathrm{kg}$

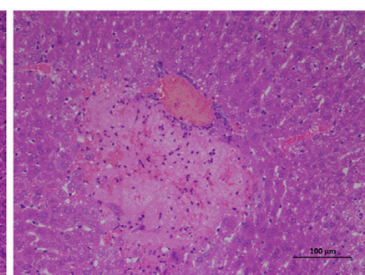

ANIT

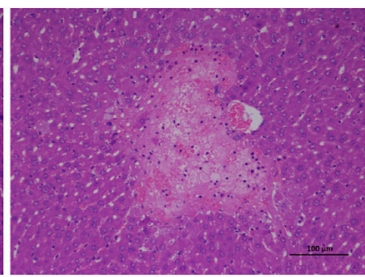

Arbutin 20mg/kg

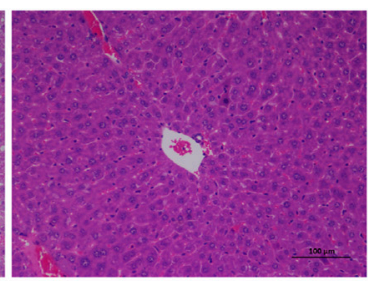

UDCA

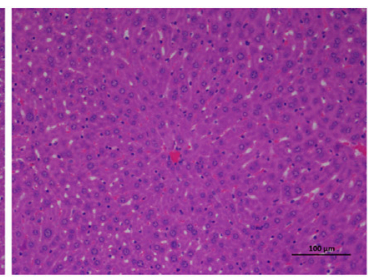

Arbutin $40 \mathrm{mg} / \mathrm{kg}$

FIGURE 3 | Effects of arbutin on histological or morphologic variations in ANIT-induced cholestasis mouse. (I) Liver tissues by H\&E stained ( $\times 200)$.

TABLE 3 | Effect of arbutin on morphological changes of the liver after ANIT treatment. Data are expressed as the mean of six specimens for each group. -, negative; +, mild (<10 cells).

\begin{tabular}{|c|c|c|c|c|}
\hline Group & Dose (mg/kg) & $\begin{array}{c}\text { Hepatocyte } \\
\text { hydropic degeneration }\end{array}$ & Hepatocellular necrosis & $\begin{array}{l}\text { Inflammatory } \\
\text { cell infiltration }\end{array}$ \\
\hline Control & - & - & - & - \\
\hline ANIT & 75 & +++ & +++ & +++ \\
\hline UDCA & 30 & - & - & - \\
\hline Arbutin (low) & 10 & ++ & ++ & ++ \\
\hline Arbutin (medium) & 20 & + & + & + \\
\hline Arbutin (high) & 40 & - & - & - \\
\hline
\end{tabular}

by Western blot or real-time PCR. In our results, ANIT treatment attenuated the transcriptional levels of basolateral uptake transporters, Oatp1b2 as well as Ntcp, which was restored by UDCA or arbutin. The mRNA levels of BSEP and Mrp2, two canalicular efflux transporters, were decreased by ANIT, but arbutin could rescue Bsep, rather than Mrp2 (Figure 4B). For the BA transporters, the mRNA levels of Mrp3 and Mrp4 were reduced in ANIT-treated mice, which could not be affected by arbutin. Furthermore, arbutin reversed the reduced transcriptional levels of Cyp7a1 or Cyp8b1, bile acid synthesis enzymes, caused by ANIT. The levels of Cyp7a1 and Cyp8b1 also decreased when treated with UDCA. Meanwhile, UDCA and arbutin both could restore the expressions of bile acid detoxification enzymes, Sult2a1 as well as Ugt1a1, which are attenuated by ANIT (Figure 4). Altogether, arbutin can reverse the altered several genes expression related to BAs homeostasis.

\section{Arbutin Activates the FXR-Associated Pathways}

As the biosynthesis or transport of bile acid is controlled by the FXR signaling pathway, we analyzed the expression levels of FXR and downstream proteins, Cyp8b1, Bsep, and Ugtla1 by Western blot. As expected, the suppressed expression of FXR in ANITtreated mice was restored by UDCA and arbutin.

We also observed that the downstream biosynthetic enzymes (Cyp8b1, Cyp7a1, and Ugtla1) and transporters (Bsep, Ntcp, and Oatp1b2) of bile acid could be reversed by arbutin treatment in a dose-dependent way (Figures $\mathbf{5 A - C}$ ). To further validate whether arbutin could be a potential agonist for FXR, we transfected with siRNA to knockdown the FXR in L-02 cells; we then analyzed the levels of the FXR by Western blot and fluorescence absorbance (Figures 5D,E). Together, these results indicated that the FXR might have participated in arbutin regulated genes involved in BAs metabolism, suggesting arbutin may be a potential and efficient agonist for FXR to exert the hepatoprotection against cholestasis caused by ANIT.

\section{Molecular Docking Simulations Implicate That Arbutin May Be a Potential Agonist of FXR}

To confirm that arbutin has potential to bind to the FXR, molecular docking analysis with arbutin and the residues from the NR1H4 3D structure (code: 4QE6) was performed. Obeticholic acid (OCA) is a well-known potent agonist of the FXR and docked with a score of $-11.1 \mathrm{~kJ} / \mathrm{mol}$ (Supplementary Figure S1B) (Zhang et al., 2020). 
A

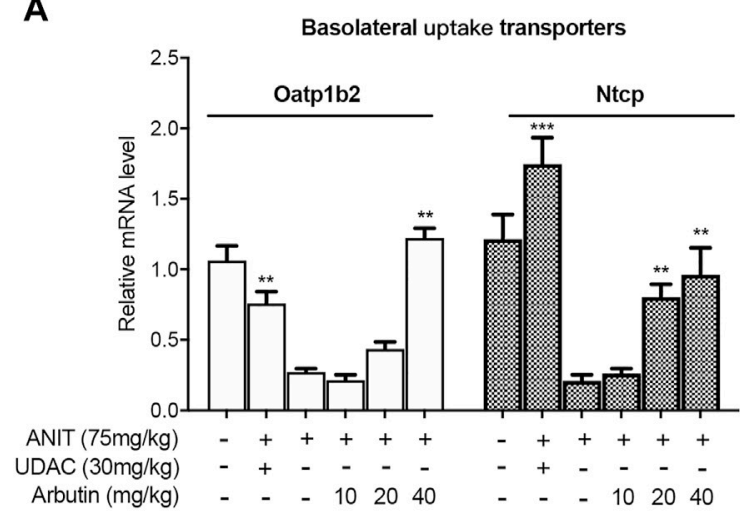

C

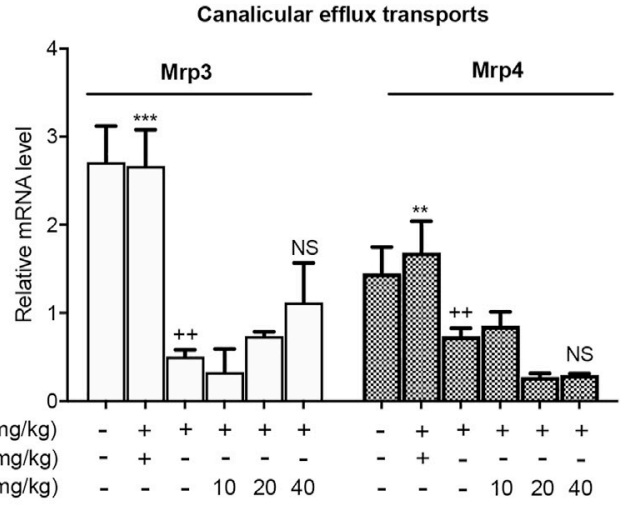

B

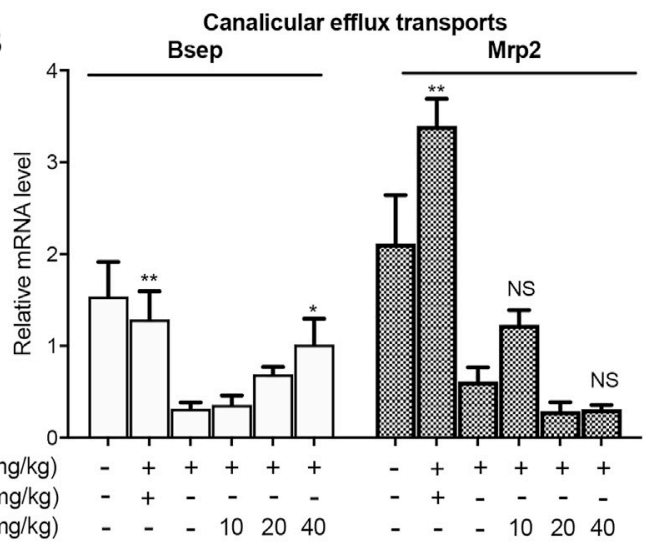

D

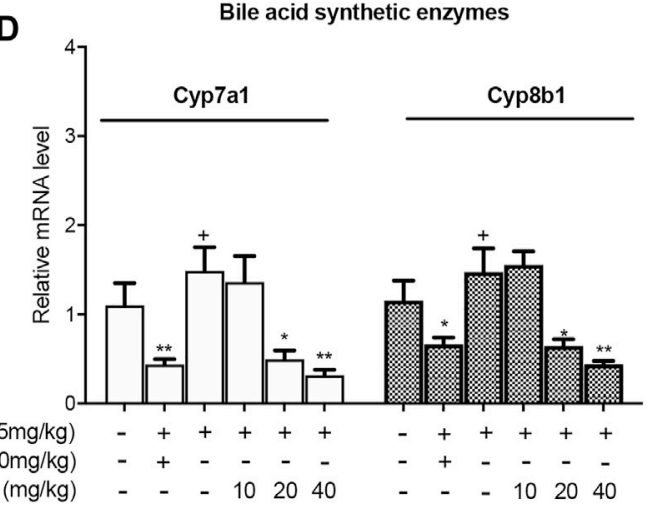

E

Bile acid detoxific enzymes

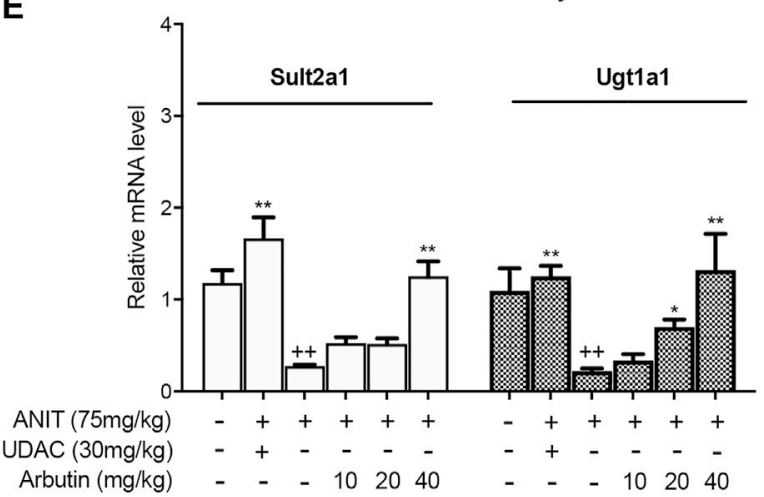

FIGURE 4 | Arbutin changed hepatic genes related to BA metabolism in mouse. Real-time PCR was performed to analyze the levels of genes related to BA metabolism including Oatp1b2, Ntcp, Bsep, Mrp2, Mrp3, Mrp4, Cyp7a1, Cyp8b1, Sult2a1, and Ugt1a1 (A-E). Data are expressed as mean \pm SD ( $n=3$ ). $p<0.05$; $+p<$ 0.05 vs. control; ${ }^{\star} p<0.05$ vs. model.

Similar to the interaction between the FXR and OCA, the energy for binding between arbutin and FXR was $-7.4 \mathrm{~kJ} / \mathrm{mol}$, implicating that the interaction was stable (Figure 6A). As shown in results, arbutin binds to a hydrophobic pocket consisting of amino acid residues (Met328, Ser332, Ile335, Arg331, His294, Met290, Ala291, Leu287, Phe336, Phe366, Met365, Ile362, Tyr369, Tyr361, and Ile352). Moreover, arbutin formed two essential hydrogen bonds with Met328 and Ser260, and $\pi-\pi$ interaction with Tyr369 residues of FXR, which further enhanced the bonding affinity (Figure 6B).
Together, the docking model demonstrated that arbutin could bind to the FXR, and arbutin might be a potential FXR agonist (Figure 7).

\section{DISCUSSION}

Cholestatic disease is a common pathological phenomenon in clinics caused by disordered BA homeostasis, leading to toxic gall accumulation, hepatonecrosis, and cirrhosis. ANIT is 


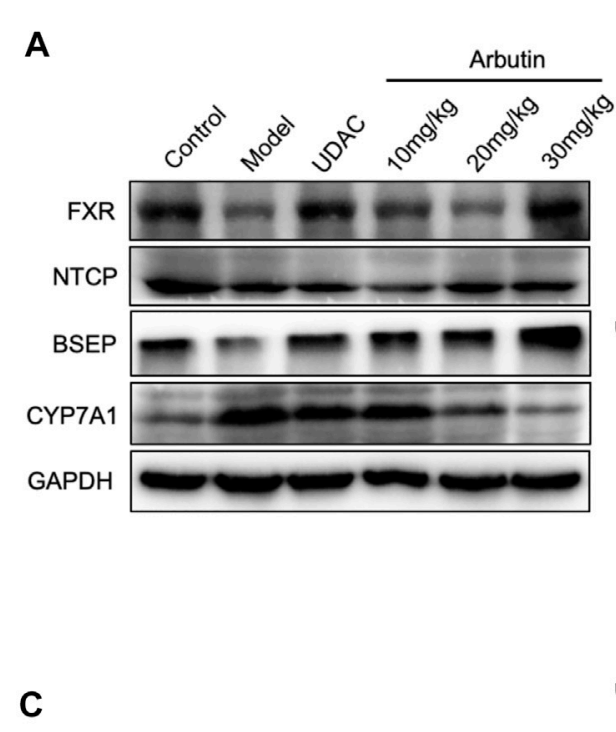

B $\quad$ FXR
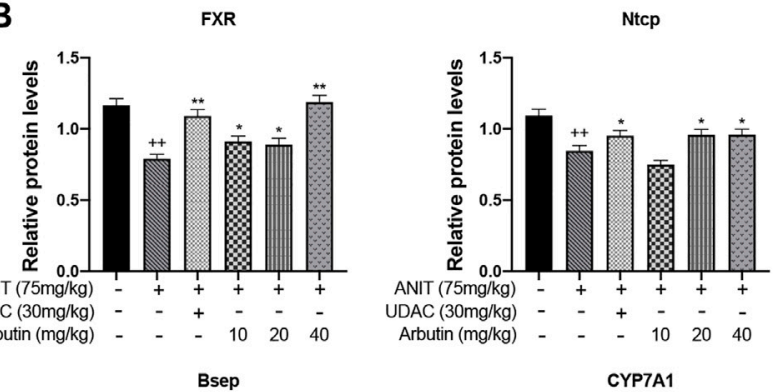

Bsep

CYP7A1

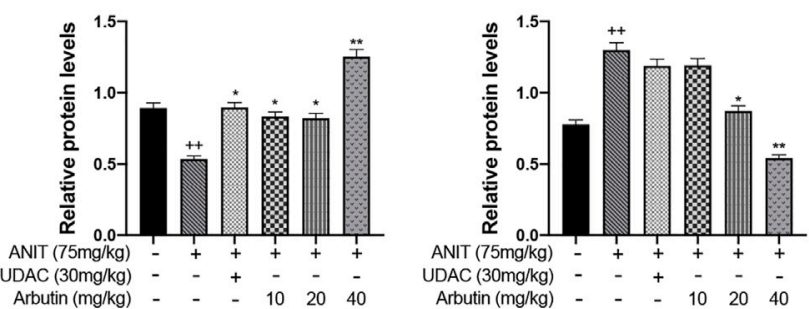

Arbutin

$(\mu \mathrm{M})$

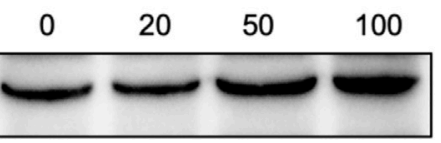

GAPDH

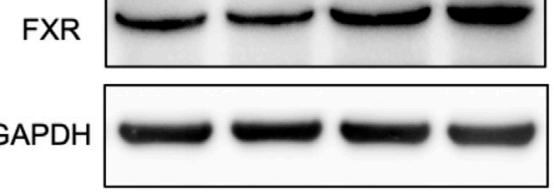

FXR

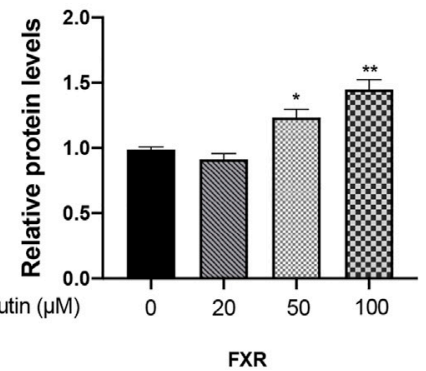

D
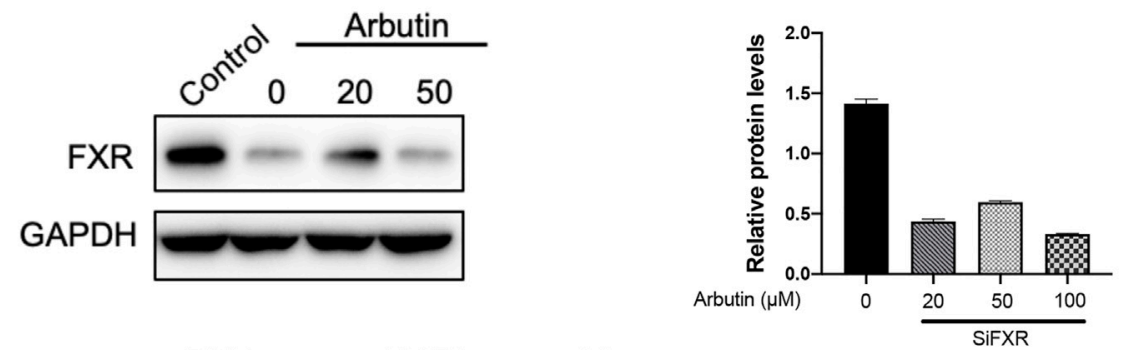

E

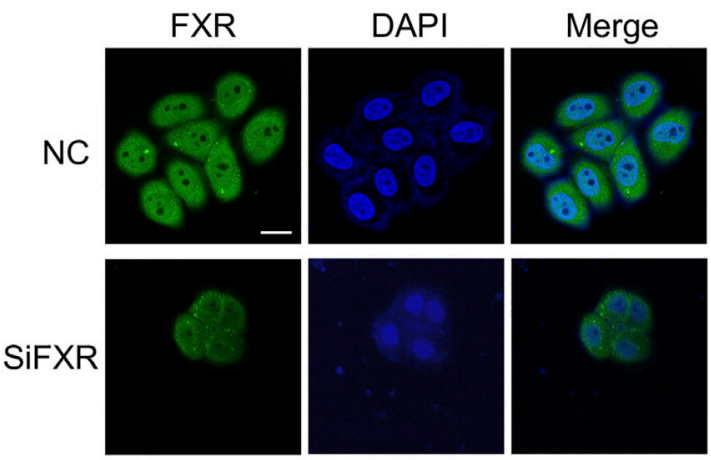

FIGURE 5 | Hepatoprotective effects of arbutin on the FXR signaling pathway in ANIT-induced cholestatic liver disease. (A) WB analysis was applied to shown protein levels of FXR, Ntcp, Bsep, and CYP7A1 with treatment indicated. (B) The analysis of the indicated proteins. (C) WB analysis was used to shown the levels of FXR in L-02 treated with different concentrates of arbutin. (D) WB analysis effects of arbutin on the expression of the FXR with or without FXR silence. (E) Immunoblotting analysis of FXR protein levels in cells after arbutin was added with or without FXR silence. 


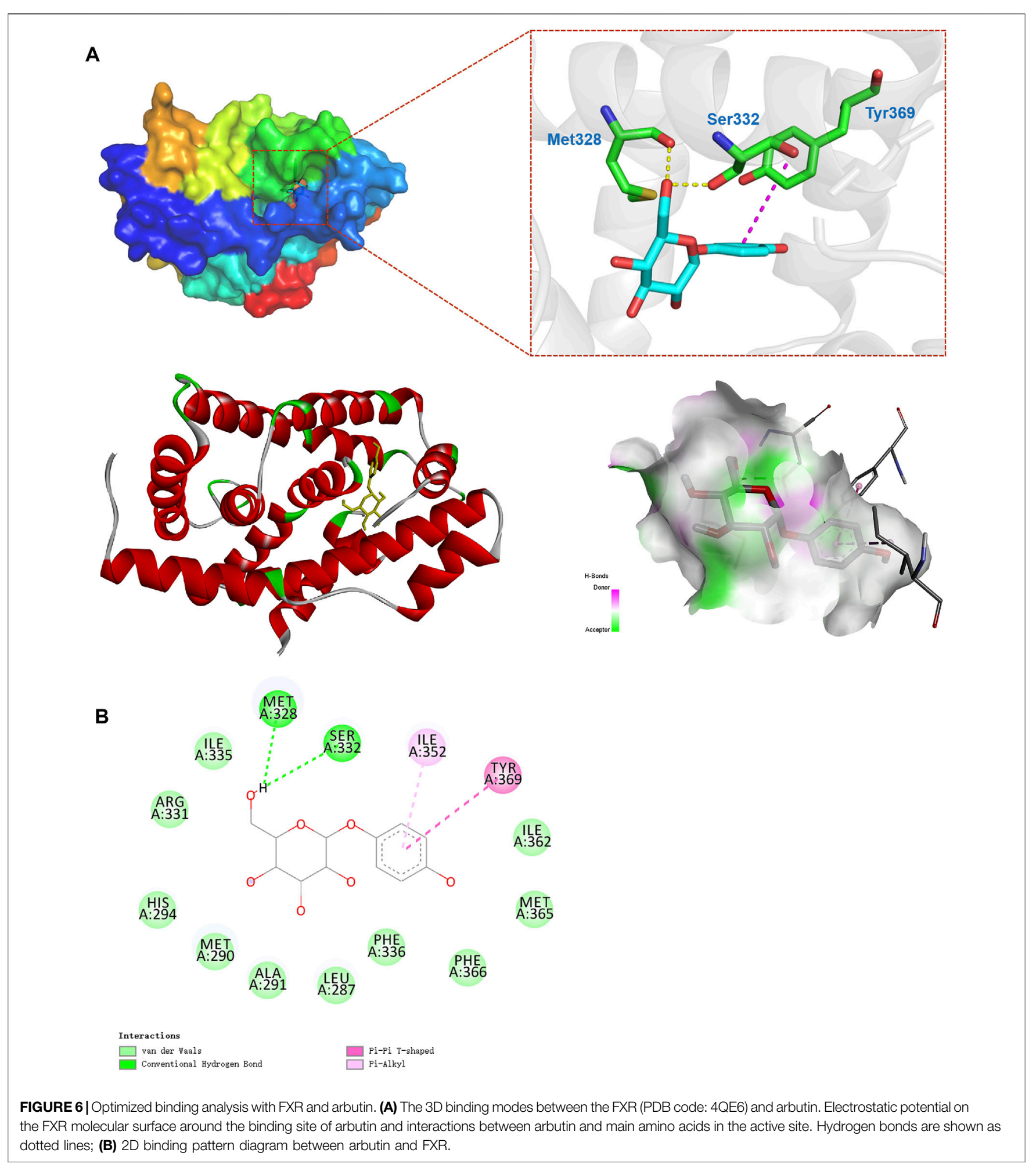

commonly used to induce cholestatic liver injury in vivo and in vitro (Mariotti et al., 2018). Recent studies also suggested ANIT-induced effects on hepatocytes, though direct injury caused by ANIT to BECs, which has been demonstrated in many studies (Carino et al., 2020; López-Riera et al., 2020).
Currently, UDCA and OCA are commonly used in clinical medicine, but these have a lot of constraints (Floreani and Mangini, 2018; Goldstein and Levy, 2018; Gao et al., 2020). Accordingly, development of appropriate therapeutic medicine for cholestasis is an urgent need. This study 


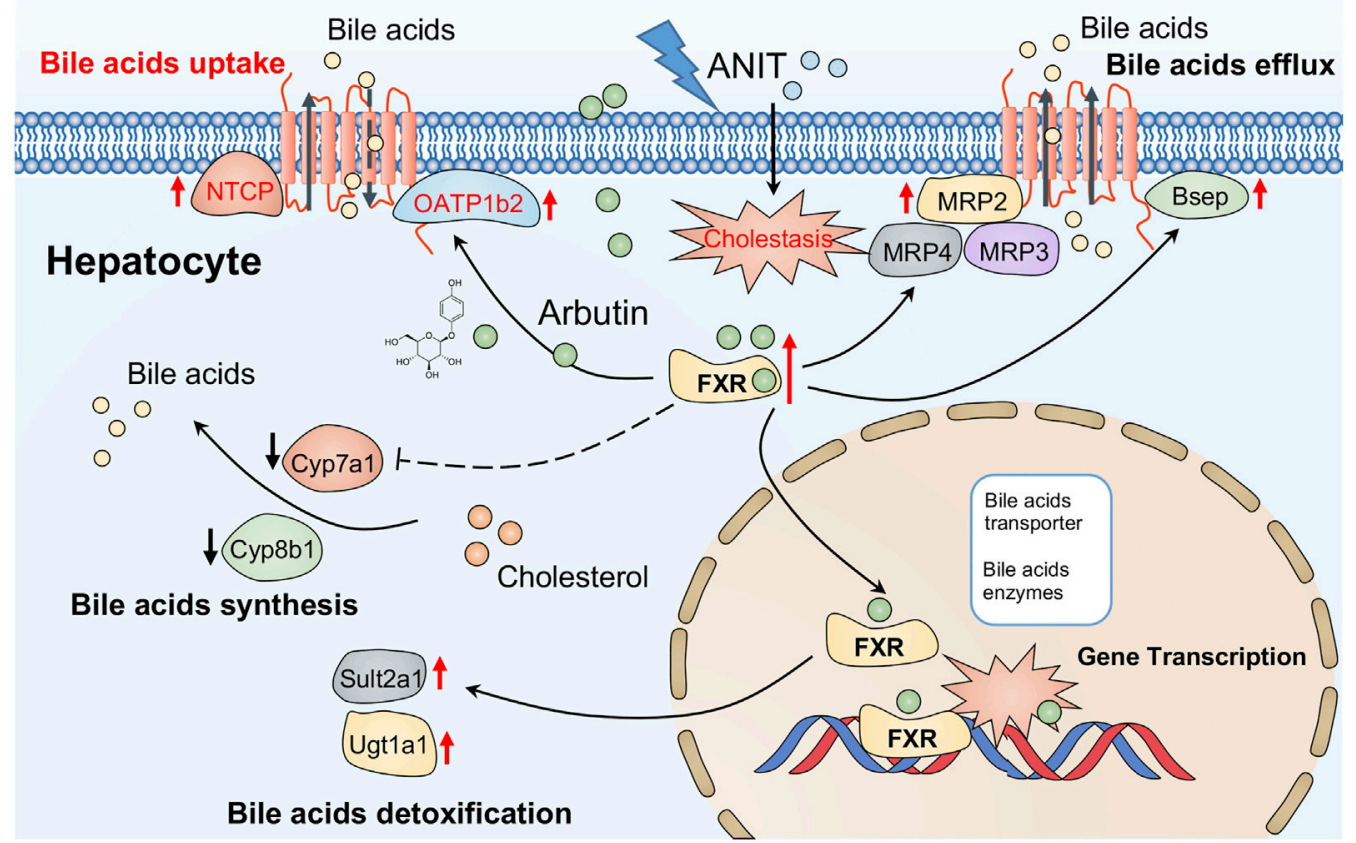

FIGURE 7 | Possible mechanism of arbutin alleviating hepatotoxicity associated with cholestasis induced by ANIT in this study.

demonstrated that arbutin had a visible hepatoprotective effect in the cholestasis model. Consistently, ANIT caused elevated serum levels of ALP, AST, ALT, $\gamma$-GT, and TBIL related to cholestasis and hepatic function (Carpenter-Deyo et al., 1991; Santamaría et al., 2019; Li et al., 2020a). These disordered serum biochemical indicators conformed to the hepatic cells and structure pattern aberration in the cholestasis mice model. Intriguingly, both arbutin and UDCA could reduce the serum indicators mentioned before. Furthermore, the deterioration of hepatic histology and morphological changes were also alleviated while arbutin and UDCA were administered.

A lot of research studies suggested that maintaining or recovery of the homeostasis of bile acid metabolism via associated transporters and enzymes may be critical for the remission of hepatotoxicity associated with cholestasis. As the homeostasis of bile acids was mainly regulated by BA transporters and synthetic enzymes, ANIT altered the expression of metabolic enzymes for bile acids to aggravate hepatic injury. The expression of Bsep, Mrp2, Ntcp, and Oatp1b2 was remarkably suppressed by ANIT. Several canalicular efflux transporters, such as Mrp2 and Bsep, regulate transportation of bile acid from hepatocytes to bile canalicular domain for bile generation (Li et al., 2016). While Oatp2 and Ntcp play a key role in reabsorption of bile acid from blood to hepatocytes. Besides, the basolateral transporters like Mrp3 and Mrp4 transport BAs from liver cells to portal blood (Keitel et al., 2019). Additionally, a series of metabolizing enzymes like Cyp8b1 and Cyp7a1 are the limiting step enzymes in BA synthesis, while Sult2al and
Ugtlal have also been demonstrated to play key roles in the detoxification of bile acid in the liver (Thakare et al., 2018). Our results suggested arbutin may restore the expression of Bsep, Ntcp, or Oatp1b2 by ANIT, while Mrp2 was slightly altered by arbutin, suggesting that Bsep, Ntcp, and Oatp1b2 may be used for bile acid transportation to protect the liver by arbutin. Mrp3 or Mrp4, as bile acid export transporter, was remarkably reduced in an adaptive responsive way caused by ANIT. While arbutin had a slight effect on Mrp4 or Mrp3, which suggested that these genes may not contribute to this process mediated by arbutin. In the recent studies, we found arbutin played at least three important roles on remission of hepatotoxicity. The first role is upregulaion of bile acid transporters via Bsep and Ntcp. The second is reduction of synthesis of bile acid through Cyp8b1 and Cyp7a1. The last role is regulation of bile acid metabolism by increasing Ugtla1 and Sult2a1.

Recent evidence has been proved that FXR is a bile acid sensor, which could control series of molecules involved in BA transport, such as Bsep, Mrps, and Ntcp. Furthermore, FXR activation increases the detoxification of bile acid by increasing Sult2a1 as well as indirectly inhibiting the synthesis of bile acid through Cyp7a1 or Cyp8b1 (Xu et al., 2003; Pathak et al., 2017; Al-Aqil et al., 2018; Chambers et al., 2019; Yu et al., 2021). Similar to previous studies, we found that the ANIT significantly inhibited the FXR and its target genes, including Ntcp, Bsep, Cyp8b1, and Ugtla1 (Jahan and Chiang, 2005; Bertaggia et al., 2017; Petrov et al., 2020). Our results have demonstrated arbutin could reverse the expression of the FXR and downstream genes in the 
cholestasis mice model, suggesting that arbutin could reduce the synthesis as well as increase transport or detoxification of bile acids by regulating the FXR. Together, we demonstrated that arbutin can attenuate the injury in ANIT-induced cholestasis and systematically investigated the possible involvement of the FXR pathway both in vitro and in vivo.

\section{CONCLUSION}

In summary, we demonstrate that arbutin can attenuate the hepatic injury in ANIT-induced cholestasis. The hepatoprotective effect of arbutin may depend on the regulation of bile acid synthetic enzymes and transporters by the activation of the FXR, suggesting that arbutin can be a potential drug to hepatic disease related to cholestasis.

\section{DATA AVAILABILITY STATEMENT}

The original contributions presented in the study are included in the article/Supplementary Material; further inquiries can be directed to the corresponding author.

\section{ETHICS STATEMENT}

The animal study was reviewed and approved by the Animal Ethics Committee of Chengdu University of Traditional Chinese Medicine.

\section{REFERENCES}

Al-Aqil, F. A., Monte, M. J., Peleteiro-Vigil, A., Briz, O., Rosales, R., González, R., et al. (2018). Interaction of Glucocorticoids with FXR/FGF19/FGF21-mediated Ileum-Liver Crosstalk. Biochim. Biophys. Acta (Bba) - Mol. Basis Dis. 1864(9 Pt B), 2927-2937. doi:10.1016/j.bbadis.2018.06.003

Alrefai, W. A., and Gill, R. K. (2007). Bile Acid Transporters: Structure, Function, Regulation and Pathophysiological Implications. Pharm. Res. 24(10), 1803-1823. doi:10.1007/s11095-007-9289-1

Bertaggia, E., Jensen, K. K., Castro-Perez, J., Xu, Y., Di Paolo, G., Chan, R. B., et al. (2017). Cyp8b1 Ablation Prevents Western Diet-Induced Weight Gain and Hepatic Steatosis Because of Impaired Fat Absorption. Am. J. PhysiologyEndocrinology Metab. 313(2), E121-e133. doi:10.1152/ajpendo.00409.2016

Carino, A., Biagioli, M., Marchianò, S., Fiorucci, C., Bordoni, M., Roselli, R., et al. (2020). Opposite Effects of the FXR Agonist Obeticholic Acid on Mafg and Nrf2 Mediate the Development of Acute Liver Injury in Rodent Models of Cholestasis. Biochim. Biophys. Acta (Bba) - Mol. Cel Biol. Lipids 1865(9), 158733. doi:10.1016/j.bbalip.2020.158733

Carpenter-Deyo, L., Marchand, D. H., Jean, P. A., Roth, R. A., and Reed, D. J. (1991). Involvement of Glutathione in 1-naphthylisothiocyanate (ANIT) Metabolism and Toxicity to Isolated Hepatocytes. Biochem. Pharmacol. 42(11), 2171-2180. doi:10.1016/0006-2952(91)90353-7

Chambers, K. F., Day, P. E., Aboufarrag, H. T., and Kroon, P. A. (2019). Polyphenol Effects on Cholesterol Metabolism via Bile Acid Biosynthesis, CYP7A1: A Review. Nutrients 11(11, 2588). doi:10.3390/nu11112588

Corpechot, C., Chazouillères, O., Belnou, P., Montano-Loza, A. J., Mason, A., Ebadi, M., et al. (2020). Long-term Impact of Preventive UDCA Therapy after Transplantation for Primary Biliary Cholangitis. J. Hepatol. 73(3), 559-565. doi:10.1016/j.jhep.2020.03.043

\section{AUTHOR CONTRIBUTIONS}

BL and PW conceptualized the study. PW, BL, and HY searched the literature and wrote the manuscript, and LQ contributed to the figures. BL, CL, HM, and WW edited and made significant revisions to the manuscript. All authors read and approved the final manuscript.

\section{FUNDING}

This work was supported by grants from the China Postdoctoral Science Foundation (2021M693784), the Science and Technology Project of Sichuan Province (2020YFS0333), and Special Postdoctoral Science Program of Sichuan Province, Potential Postdoctoral Program of Chengdu University of Traditional Chinese Medicine (BSH2019019).

\section{ACKNOWLEDGMENTS}

We thank the collaborators for their contributions to the publications cited in this study.

\section{SUPPLEMENTARY MATERIAL}

The Supplementary Material for this article can be found online at: https://www.frontiersin.org/articles/10.3389/fcell.2021.758632/ full\#supplementary-material

Dong, R., Yang, X., Wang, C., Liu, K., Liu, Z., Ma, X., et al. (2019). Yangonin Protects against Non-alcoholic Fatty Liver Disease through Farnesoid X Receptor. Phytomedicine 53, 134-142. doi:10.1016/j.phymed.2018.09.006

Dyson, J. K., and Jones, D. E. J. (2020). UDCA Prophylaxis for post-transplant PBC Recurrence Prevention: Time to Change Practice. J. Hepatol. 73(3), 499-501. doi:10.1016/j.jhep.2020.04.040

Feldman, A. G., and Sokol, R. J. (2020). Recent Developments in Diagnostics and Treatment of Neonatal Cholestasis. Semin. Pediatr. Surg. 29(4), 150945. doi:10.1016/j.sempedsurg.2020.150945

Floreani, A., and Mangini, C. (2018). Primary Biliary Cholangitis: Old and Novel Therapy. Eur. J. Intern. Med. 47, 1-5. doi:10.1016/j.ejim.2017.06.020

Gao, L., Wang, L., Woo, E., He, X., Yang, G., Bowlus, C., et al. (2020). Clinical Management of Primary Biliary Cholangitis-Strategies and Evolving Trends. Clinic Rev. Allerg Immunol. 59(2), 175-194. doi:10.1007/s12016-019-08772-7

Garcia-Jimenez, A., Teruel-Puche, J. A., Berna, J., Rodriguez-Lopez, J. N., Tudela, J., and Garcia-Canovas, F. (2017). Action of Tyrosinase on Alpha and Beta-Arbutin: A Kinetic Study. PLoS One 12(5), e0177330. doi:10.1371/journal.pone.0177330

Goldstein, J., and Levy, C. (2018). Novel and Emerging Therapies for Cholestatic Liver Diseases. Liver Int. 38(9), 1520-1535. doi:10.1111/liv.13880

Halilbasic, E., Claudel, T., and Trauner, M. (2013). Bile Acid Transporters and Regulatory Nuclear Receptors in the Liver and beyond. J. Hepatol. 58(1), 155-168. doi:10.1016/j.jhep.2012.08.002

Hirschfield, G. M., Karlsen, T. H., Lindor, K. D., and Adams, D. H. (2013). Primary Sclerosing Cholangitis. The Lancet 382(9904), 1587-1599. doi:10.1016/s01406736(13)60096-3

Jahan, A., and Chiang, J. Y. L. (2005). Cytokine Regulation of Human Sterol $12 \alpha$ Hydroxylase (CYP8B1) Gene. Am. J. Physiology-Gastrointestinal Liver Physiol. 288(4), G685-G695. doi:10.1152/ajpgi.00207.2004

Jurica, K., Benković, V., Sikirić, S., Kopjar, N., and Brčić Karačonji, I. (2020). Liver Function and DNA Integrity in Hepatocytes of Rats Evaluated after Treatments 
with Strawberry Tree (Arbutus Unedo L.) Water Leaf Extract and Arbutin. Drug Chem. Toxicol. 43(2), 127-137. doi:10.1080/01480545.2018.1477794

Keitel, V., Dröge, C., and Häussinger, D. (2019). Targeting FXR in Cholestasis. Handb Exp. Pharmacol. 256, 299-324. doi:10.1007/164_2019_231

Kjærgaard, K., Frisch, K., Sørensen, M., Munk, O. L., Hofmann, A. F., Horsager, J., et al. (2021). Obeticholic Acid Improves Hepatic Bile Acid Excretion in Patients with Primary Biliary Cholangitis. J. Hepatol. 74(1), 58-65. doi:10.1016/j.jhep.2020.07.028

Kudlinzki, D., Merk, D., Linhard, V., Saxena, K., Schubert-Zsilavecz, M., and Schwalbe, H. (2019). "Crystal Structure of Farnesoid X Receptor (FXR) with Bound NCoA-2 Peptide and CDCA").

Kumar, P., and Kulkarni, A. (2020). UDCA Therapy in Intrahepatic Cholestasis of Pregnancy? J. Hepatol. 72(3), 586-587. doi:10.1016/j.jhep.2019.10.025

López-Riera, M., Conde, I., Castell, J. V., and Jover, R. (2020). A Novel MicroRNA Signature for Cholestatic Drugs in Human Hepatocytes and its Translation into Novel Circulating Biomarkers for Drug-Induced Liver Injury Patients. Toxicol. Sci. 173(2), 229-243. doi:10.1093/toxsci/kfz138

Li, T., and Chiang, J. Y. L. (2020). Bile Acid-Based Therapies for Non-alcoholic Steatohepatitis and Alcoholic Liver Disease. Hepatobiliary Surg. Nutr. 9(2), 152-169. doi:10.21037/hbsn.2019.09.03

Li, T., Xu, L., Zheng, R., Wang, X., Li, L., Ji, H., et al. (2020a). Picroside II Protects against Cholestatic Liver Injury Possibly through Activation of Farnesoid X Receptor. Phytomedicine 68, 153153. doi:10.1016/j.phymed.2019.153153

Li, T., Zheng, R., Xu, L., Zhou, M., Wang, X., Guo, Q., et al. (2020b). Picroside II Alleviates Liver Injury Induced by Alpha-Naphthylisothiocyanate through AMPK-FXR Pathway. Toxicol. Appl. Pharmacol. 408, 115248. doi:10.1016/j.taap.2020.115248

Li, W.-K., Wang, G.-F., Wang, T.-M., Li, Y.-Y., Li, Y.-F., Lu, X.-Y., et al. (2019). Protective Effect of Herbal Medicine Huangqi Decoction against Chronic Cholestatic Liver Injury by Inhibiting Bile Acid-Stimulated Inflammation in DDC-Induced Mice. Phytomedicine 62, 152948. doi:10.1016/j.phymed.2019.152948

Li, X., Liu, R., Yu, L., Yuan, Z., Sun, R., Yang, H., et al. (2016). Alphanaphthylisothiocyanate Impairs Bile Acid Homeostasis through AMPK-FXR Pathways in Rat Primary Hepatocytes. Toxicology 370, 106-115. doi:10.1016/ j.tox.2016.09.020

Lindpaintner, E. (1939). Arbutin und Methylarbutin und ihre Bestimmung in Drogen. Mit Unterstützung der Deutschen Forschungsgemeinschaft. Arch. Pharm. Pharm. Med. Chem. 277(9), 398-415. doi:10.1002/ardp.19392770903

Mariotti, V., Strazzabosco, M., Fabris, L., and Calvisi, D. F. (2018). Animal Models of Biliary Injury and Altered Bile Acid Metabolism. Biochim. Biophys. Acta (Bba) - Mol. Basis Dis. 1864(4 Pt B), 1254-1261. doi:10.1016/j.bbadis.2017.06.027

Meier, P. J., and Stieger, B. (2002). Bile Salt Transporters. Annu. Rev. Physiol. 64, 635-661. doi:10.1146/annurev.physiol.64.082201.100300

Migas, P., and Krauze-Baranowska, M. (2015). The Significance of Arbutin and its Derivatives in Therapy and Cosmetics. Phytochemistry Lett. 13, 35-40. doi:10.1016/j.phytol.2015.05.015

Mirshahvalad, S., Feizi, F., Barkhordar, A., Bahadoram, M., Houshmand, G., and Pouramir, M. (2016). Hepatoprotective Effects of Arbutin against Liver Damage Induced by Carbon Tetrachloride in Rats. Jundishapur J. Nat. Pharm. Prod. 11(3), e33392. doi:10.17795/jinpp.33392

Nadi, S., Elahi, M., Moradi, S., Banaei, A., Ataei, G., and Abedi-Firouzjah, R. (2019). Radioprotective Effect of Arbutin in Megavoltage Therapeutic X-Irradiated Mice Using Liver Enzymes Assessment. J. Biomed. Phys. Eng. 9(5), 533-540. doi:10.31661/jbpe.v0i0.1199

Nakanishi, Y., and Saxena, R. (2015). Pathophysiology and Diseases of the Proximal Pathways of the Biliary System. Arch. Pathol. Lab. Med. 139(7), 858-866. doi:10.5858/arpa.2014-0229-RA

Navaneethan, U., Spencer, C., Zhu, X., Vargo, J. J., Grove, D., and Dweik, R. A. (2021). Volatile Organic Compounds in Bile Can Distinguish Pancreatic Cancer from Chronic Pancreatitis: a Prospective Observational Study. Endoscopy 53(7), 732-736. doi:10.1055/a-1255-9169

Ocvirk, S., and O'Keefe, S. J. D. (2021). Dietary Fat, Bile Acid Metabolism and Colorectal Cancer. Semin. Cancer Biol. 73, 347-355. doi:10.1016/ j.semcancer.2020.10.003

Ortiz-Ruiz, C. V., Garcia-Molina, M. D. M., Serrano, J. T., Tomas-Martinez, V., and Garcia-Canovas, F. (2015). Discrimination between Alternative Substrates and Inhibitors of Tyrosinase. J. Agric. Food Chem. 63(8), 2162-2171. doi:10.1021/jf5051816

Pathak, R. D., Schroeder, E. B., Seaquist, E. R., Zeng, C., Lafata, J. E., Thomas, A., et al. (2017). Erratum. Response to Comment on Pathak et al. Severe
Hypoglycemia Requiring Medical Intervention in a Large Cohort of Adults With Diabetes Receiving Care in U.S. Integrated Health Care Delivery Systems: 2005-2011. Diabetes Care 2016;39:363-370. Diabetes Care 2017;40:e26. Dia Care 2016;39:1-1420. Diabetes Care 2017;40:e26. Diabetes Care 40(10), 1420. doi:10.2337/dc17-er10

Petrov, P. D., Fernández-Murga, L., Conde, I., Martínez-Sena, T., Guzmán, C., Castell, J. V., et al. (2020). Epistane, an Anabolic Steroid Used for Recreational Purposes, Causes Cholestasis with Elevated Levels of Cholic Acid Conjugates, by Upregulating Bile Acid Synthesis (CYP8B1) and Cross-Talking with Nuclear Receptors in Human Hepatocytes. Arch. Toxicol. 94(2), 589-607. doi:10.1007/ s00204-019-02643-y

Rimland, C. A., Tilson, S. G., Morell, C. M., Tomaz, R. A., Lu, W. Y., Adams, S. E., et al. (2021). Regional Differences in Human Biliary Tissues and Corresponding In Vitro -Derived Organoids. Hepatology 73(1), 247-267. doi:10.1002/ hep. 31252

Santamaría, E., Rodríguez-Ortigosa, C. M., Uriarte, I., Latasa, M. U., Urtasun, R., Alvarez-Sola, G., et al. (2019). The Epidermal Growth Factor Receptor Ligand Amphiregulin Protects from Cholestatic Liver Injury and Regulates Bile Acids Synthesis. Hepatology 69(4), 1632-1647. doi:10.1002/hep.30348

Stofan, M., and Guo, G. L. (2020). Bile Acids and FXR: Novel Targets for Liver Diseases. Front. Med. (Lausanne) 7, 544. doi:10.3389/fmed.2020.00544

Tang, H. C., and Chen, Y. C. (2015). Identification of Tyrosinase Inhibitors from Traditional Chinese Medicines for the Management of Hyperpigmentation. Springerplus 4, 184. doi:10.1186/s40064-015-0956-0

Thakare, R., Alamoudi, J. A., Gautam, N., Rodrigues, A. D., and Alnouti, Y. (2018). Species Differences in Bile Acids I. Plasma and Urine Bile Acid Composition. J. Appl. Toxicol. 38(10), 1323-1335. doi:10.1002/jat.3644

Wu, P., Gao, W., Su, M., Nice, E. C., Zhang, W., Lin, J., et al. (2021). Adaptive Mechanisms of Tumor Therapy Resistance Driven by Tumor Microenvironment. Front Cel Dev Biol 9, 641469. doi:10.3389/fcell.2021.641469

Xu, G., Li, H., Pan, L. X., Shang, Q., Honda, A., Ananthanarayanan, M., et al. (2003). FXR-mediated Down-Regulation of CYP7A1 Dominates LXRalpha in Long-Term Cholesterol-Fed NZW Rabbits. J. Lipid Res. 44(10), 1956-1962. doi:10.1194/jlr.M300182-JLR200

Xu, W. H., Liang, Q., Zhang, Y. J., and Zhao, P. (2015). Naturally Occurring Arbutin Derivatives and Their Bioactivities. Chem. Biodivers 12(1), 54-81. doi:10.1002/cbdv.201300269

Yu, L., Lu, H., Yang, X., Li, R., Shi, J., Yu, Y., et al. (2021). Diosgenin Alleviates Hypercholesterolemia via SRB1/CES-1/CYP7A1/FXR Pathway in High-Fat Diet-Fed Rats. Toxicol. Appl. Pharmacol. 412, 115388. doi:10.1016/ j.taap. 2020.115388

Zhang, G., Sun, X., Wen, Y., Shi, A., Zhang, J., Wei, Y., et al. (2020). Hesperidin Alleviates Cholestasis via Activation of the Farnesoid X Receptor In Vitro and In Vivo. Eur. J. Pharmacol. 885, 173498. doi:10.1016/j.ejphar.2020.173498

Zhou, H., Zhao, J., Li, A., and Reetz, M. T. (2019). Chemical and Biocatalytic Routes to Arbutin (†). Molecules 24(18). doi:10.3390/molecules24183303

Zollner, G., and Trauner, M. (2008). Mechanisms of Cholestasis. Clin. Liver Dis. 12(1), 1-26. vii. doi:10.1016/j.cld.2007.11.010

Zollner, G., and Trauner, M. (2006). Molecular Mechanisms of Cholestasis. Wien Med. Wochenschr 156(13-14), 380-385. doi:10.1007/s10354-006-0312-7

Conflict of Interest: The authors declare that the research was conducted in the absence of any commercial or financial relationships that could be construed as a potential conflict of interest.

Publisher's Note: All claims expressed in this article are solely those of the authors and do not necessarily represent those of their affiliated organizations, or those of the publisher, the editors, and the reviewers. Any product that may be evaluated in this article, or claim that may be made by its manufacturer, is not guaranteed or endorsed by the publisher.

Copyright $\odot 2021 \mathrm{Wu}$, Qiao, Yu, Ming, Liu, Wu and Li. This is an open-access article distributed under the terms of the Creative Commons Attribution License (CC $B Y)$. The use, distribution or reproduction in other forums is permitted, provided the original author(s) and the copyright owner(s) are credited and that the original publication in this journal is cited, in accordance with accepted academic practice. No use, distribution or reproduction is permitted which does not comply with these terms. 